\title{
NEAR-RINGS WITH CHAIN CONDITIONS ON RIGHT ANNIHILATORS
}

\author{
by A. OSWALD \\ (Received 29th September 1978)
}

Throughout this note, $N$ wili denote a (Left) near-ring with two-sided zero. Definitions of basic concepts can be found in (9).

We prove first that a right ideal $I$ in a d.g. near-ring has a right identity if and only if $x \in x I$ for each $x \in I$. This enables us to study the structure of regular d.g. near-rings with chain conditions on right annihilators. Specifically we will prove that a regular d.g. near-ring with both the maximum and the minimum conditions on right annihilators is a finite direct sum of near-rings which are either rings of matrices over division rings or non-rings of the form $M_{G}(\Gamma)$ for a suitable type $2 N$-module $\Gamma$. Finally we consider the case of maximum condition on $N$-subgroups.

These results generalise some results of Heatherly (5).

The idea for our first result is taken from (4).

Theorem 1. Let $N$ be a d.g. near-ring and $I$ be a right ideal of $N$ which, as a near-ring, has the minimum condition on right annihilators. Then the near-ring I has $a$ right identity if and only if $x \in x I$ for each $x \in I$.

Proof. The necessity is immediate. Let $N$ be d.g. by $S$ and for $a \in I$ define $L_{a}=\{s-a s: s \in S\}$. Choose $e \in I$ such that $l_{I}\left(L_{e}\right)=\left\{x \in I: x L_{e}=(0)\right\}$ is maximal where $b \in l_{I}\left(L_{e}\right)$ if and only if $b L_{e}=(0)$ and $b \in I$. Suppose $l_{I}\left(L_{e}\right) \neq I$. Choose $y \in I$ with $y L_{e} \neq(0)$ and $s \in S$ with $y(s-e s) \neq 0$. Then $(y-y e) s \neq 0$ so $y-y e \neq 0$. Also $y-y e \in$ $(y-y e) I$ so $y-y e=(y-y e) e^{\prime}$ for some $e^{\prime} \in I$. Writing $e^{\prime}=\Sigma \pm s_{i}$ where $s_{i} \in S$ we then have

$$
\begin{aligned}
y & =(y-y e) e^{\prime}+y e \\
& =y\left(\sum \pm\left(s_{i}-e s_{i}\right)+e\right) \\
& =y\left(\sum \pm s_{i}+u+e\right) \text { for some } u \in I \\
& =y f, \text { where } f=e^{\prime}+u+e .
\end{aligned}
$$

Now $y \in l_{I}\left(L_{f}\right)$ but $y \notin l_{I}\left(L_{e}\right)$. Also $z \in l_{I}\left(L_{e}\right)$ implies $z f=z\left(\Sigma \pm\left(s_{i}-e s_{i}\right)+e\right)=z e$ and so $z \in l_{I}\left(L_{f}\right)$. This contradicts the maximality of $l_{I}\left(L_{e}\right)$ and so $l_{I}\left(L_{e}\right)=I$. But then if $r \in I$ we have $r L_{e}=(0)$ and so $(r-r e) S=(0)$ from which $(r-r e) N=(0)$. In particular $(r-r e) I=(0)$ and thus $r=r e$ and $e$ is a right identity. 
Corollary 1. A d.g. near-ring with minimum condition on right annihilators has a right identity if and only if $x \in x N$ for each $x \in N$.

As a first application of this theorem we give an alternative proof of a theorem of Szeto (11). We say that a near-ring $N$ is a subdirect sum of near-rings $N_{\lambda}$ if and only if there exist ideals $I_{\lambda}$ of $N$ with $\cap_{\lambda} I_{\lambda}=(0)$ and $N_{\lambda} \approx N / I$ as near-rings. Then as in Stewart (10) we have.

Lemma 1. A near-ring $N$ has no nilpotent elements if and only if it is isomorphic to a subdirect sum of near-rings without proper divisors of zero.

Lemma 2. (Beidleman (1)). If $N$ is a regular near-ring and $0 \neq b \in N$ then $b N=f N$ for some idempotent $f \in N$.

Theorem 2. (Szeto (11)). A regular d.g. near-ring $N$ has no nilpotent elements if and only if it is a subdirect sum of division near-rings.

Proof. The sufficiency is immediate. Suppose $N$ has no nilpotent elements. Then by Lemma 1 it is a subdirect sum of near-rings without proper divisors of zero each of which is d.g., regular and trivially has the minimum condition on right annihilators. Let $N_{1}$ be one such subdirect summand. From Corollary 1 it has a right identity, $e$ say. Then $0 \neq x \in N_{1}$ implies $x N_{1}=f N_{1}$ for some idempotent $f \in N_{1}$. Since $r(f)=$ $\left\{x \in N_{1}: f x=0\right\}=(0)$ we get $N_{1}=f N_{1}=x N_{1}$ and so $e=x y$ for some $y \in N_{1}$. Thus $N_{1} \mid\{0\}$ is a group and $N_{1}$ is a division near-ring as required.

Observe that since $N_{1}$ is a division near-ring then by Ligh (7) it has abelian addition. Consequently from Fröhlich $(3 ; 4.4 .1)$ it is distributive and hence is a ring. We thus get

Corollary 2. A regular d.g. near-ring has no (non-zero) nilpotent elements if and only if it is a ring and a subdirect sum of division rings.

If $I$ is an ideal of the near-ring $N$ and $x \in I$ we denote by $S g_{I}(x)\left(S g_{N}(x)\right)$ the $I$-subgroup ( $N$-subgroup) of $I(N)$ generated by $x$. Clearly $S g_{N}(x) \subseteq I$ and $S g_{I}(x) \subseteq$ $S g_{N}(x)$.

Theorem 3. Let $N$ be d.g. and I be an ideal of $N$ which, as a near-ring, has the minimum condition on right annihilators and $A^{2}=A$ for each $I$-subgroup $A$ of $I$. Then $I$ has an identity which is a central idempotent of $N$.

Proof. If $L$ is an $I$-subgroup of $I$ and $x \in L$ then $x N$ is an $I$-subgroup of $I$. Also $x N=x N x N \subseteq x I \subseteq L$ so each $I$-subgroup of $I$ is an $N$-subgroup of $N$ contained in $I$. Hence if $x \in I$ then $S g_{N}(x) \subseteq S g_{I}(x)$ and thus $S g_{I}(x)=S g_{N}(x)$. Now $x \in S g_{N}(x)=$ $S g_{N}(x) S g_{N}(x)$ and so $x=u v$ for some $u, v \in S g_{N}(x)$. If $N$ is distributively generated by $S$ then $v=\Sigma \pm s_{i}$ where $s_{i} \in S$ and $u=\Sigma x r_{j}$ where $r_{j} \in N$ or $r_{j}$ is formal identity. Hence $u v=\Sigma x r_{j} \Sigma \pm s_{i}=x \Sigma \pm r_{j} s_{i} \subseteq x N=x N x N \subseteq x I$. Applying Theorem 1 we see that $I$ has a right identity $e$. Now suppose that $y \in N$ with $z=e y-y e$. Then $z \in I$ and 
$e z=e y-e(y e)=0$ from which $I e z I=(0)$ and $I z I=(0)$. Then $(z I)^{2}=(0)$ so that $z I=(0)$ and $z=0$. Thus $e$ is a central idempotent of $N$ and a two-sided identity for $I$.

Corollary 3. If $N$ is a regular, d.g. near-ring with the minimum condition on right annihilators then $N$ has a two-sided identity.

Proof. $N$ regular implies $A^{2}=A$ for each $N$-subgroup $A$ of $N$.

Corollary 4. A d.g. near-ring with the minimum condition on $N$-subgroups and no nilpotent $N$-subgroups has a two-sided identity.

Proof. Such a near-ring is completely reducible (8) and hence regular.

Theorem 4. A d.g. near-ring $N$ which is regular with the minimum condition on right annihilators is a direct sum of ideals which are simple d.g. near-ring with identity.

Proof. If $I$ is a non-zero ideal of $N$ then $I$ has an identity $e$ and so $I \cap r(e)=(0)$. Now $N=I \oplus r(e)$ and if $x \in r(e)$ and $n \in N$ then $e n x=n e x=0$ so that $r(e)$ is an ideal of $N$ and hence has a two-sided identity $f$. Now $I \subseteq r(f)$ and $r(f) \cap r(e)=(0)$ from which $I=r(f)$. It follows that every ideal of $N$ is of the form $r(f)$ for some central idempotent $f$ of $N$. Let $r\left(e_{1}\right)$ be a minimal non-zero ideal of $N$. There is an ideal of $N, I_{1}$, with $I_{1} \cap r\left(e_{1}\right)=(0)$ and $I_{1} \oplus r\left(e_{1}\right)=N$. Choose $e_{2} \in I_{1}$ with $r\left(e_{2}\right)$ a minimal non-zero ideal of $N$ in $I_{1}$. For some ideal $I_{2}$ of $N$ we have $N=$ $I_{2} \oplus r\left(e_{1}\right) \oplus r\left(e_{2}\right)$. In this way we construct a descending chain $I_{1} \supseteq I_{2} \supseteq \ldots$ of right annihilators. It follows that for some $k, N=r\left(e_{1}\right) \oplus \ldots \oplus r\left(e_{k}\right)$. If $I$ is an ideal of $N$ and if $B$ is an ideal of $I$ then $B N \subseteq B$ when $I$ is a direct summand of $N$. Furthermore, if $e$ is the identity of $I$ then $e N=N e$ and $N B=N e B=e N B \subseteq I B \subseteq B$. Hence the ideals $r\left(e_{i}\right)$ are simple and we have the result.

The following result is proven in the same way as in Koh (6).

Lemma 3. If $N$ is regular and $I$ is a maximal annihilator right ideal of $N$ then there is a minimal $N$-subgroup $B=e N$, where $e$ is an idempotent, with $I \cap B=(0)$ and $I+B=N$.

An $N$-module $\Gamma$ is type-2 if $\Gamma N \neq(0)$ and $\Gamma$ has no proper $N$-subgroups. If $N$ has an identity then $\Gamma$ has no proper $N$-subgroups if and only if $\gamma N=\Gamma$ or $\gamma N=(0)$ for each $\gamma \in \Gamma$. Hence if $N$ is a regular near-ring with the maximum condition on right annihilators then $N$ has an $N$-subgroup which is a type- $2 N$-module. If, in addition, $N$ has no non-trivial two-sided ideals this $N$-subgroup $\Gamma$ will be such that $\Gamma a=(0)$ implies that $a=0$ and $\Gamma$ will be a type-2 faithful $N$-module. In such a case we say that $N$ is 2-primitive on $\Gamma$.

In the case where $N$ is a ring $\Gamma$ will be a faithful ring module so that $N$ will be a regular simple primitive ring and if $N$ also has the minimum condition on right annihilators then $N$ will have an identity. The set of minimal right ideals will be non-empty and the sum of all of them will be an ideal containing $\Gamma$ and hence will be 
$N$. It follows that $N$ will be the sum of finitely many minimal right ideals and hence $N$ will have the minimum condition on right ideals so that $N$ will be a ring of matrices over a suitable division ring.

Turning to the case where $N$ is a non-ring we have

Theorem 5. If $N$ is a non-ring with identity and the minimum condition on right annihilators which is 2-primitive on an $N$-module $\Gamma$ which is an $N$-subgroup of $N$ then $N=M_{G}(\Gamma)$ where $G=$ Aut $_{N}(\Gamma)$ and $M_{G}(\Gamma)=\{f: \Gamma \rightarrow \Gamma: f \alpha=\alpha f$ for each $\alpha \in G\}$.

Proof. This is the same as in Betsch (2; Thm 2.5) making use of the fact that since $\Gamma \subseteq N, r\left(\gamma_{1}\right) \cap r\left(\gamma_{2}\right)=r\left(\left\{\gamma_{1}, \gamma_{2}\right\}\right)$ is an annihilator right ideal of $N$.

Observe that in view of $(2 ;$ Thm 5.9$)$ we have

Corollary 5. A non-ring $N$ with identity and the minimum condition on right annihilators which is 2-primitive on an $N$-module $\Gamma$ which is an $N$-subgroup of $N$ has both the minimum and the maximum conditions on right ideals.

Theorems 4 and 5 and the intervening discussion now yield the result mentioned in the introduction.

Theorem 6. A d.g. near-ring which is regular and has the minimum and maximum conditions on right annihilators is a finite direct sum of ideals which are d.g. near-rings each of which is either a ring of matrices over a suitable division ring or a non-ring of the form $M_{G}(\Gamma)$ for a suitable type-2 near-ring module $\Gamma$.

An $N$-subgroup $A$ of $N$ is module-essential if whenever $A \cap B=(0)$ with $B$ a right ideal of $N$ then $B=(0)$. The $N$-subgroup $A$ is essential when this is true for $B$ an $N$-subgroup of $N$.

Theorem 7. Let $N$ be a near-ring in which module essential $N$-subgroups are essential. If $N$ is regular with maximum condition on right annihilators and no infinite direct sums of right ideals then $N$ has minimum condition on right annihilators.

Proof. Let $A_{1} \supset A_{2} \supset \ldots$ be a properly descending chain of right annihilators and $U$ be a left annihilator minimal subject to $l\left(A_{k}\right) \varsubsetneqq U \subseteq l\left(A_{k+1}\right)$. Then $U \neq(0)$ and $U A_{k}=(0)$. Choose $u \in U$ with $u A_{k} \neq(0)$. Since $N$ is regular, $N$ has no nilpotent $N$-subgroups so $u A_{k} u A_{k} \neq(0)$ and so for some $a \in A_{k}, u a u A_{k} \neq(0)$. If $y \in A_{k}$ with auy $\in A_{k+1} \cap a u A_{k}$ then $U a u y=(0)$. Now $l\left(A_{k}\right) \subseteq l(y)$ so $l\left(A_{k}\right) \subseteq l(y) \cap U \subseteq U$. Since $U$ is minimal either $U=l(y) \cap U$ or $l\left(A_{k}\right)=l(y) \cap U$. As uauy $=0$ we have uau $\in$ $l(y) \cap U$ whereas $u a u A_{k} \neq(0)$. It follows that $U=l(y) \cap U$ and so $U \subseteq l(y)$ and $U y=(0)$. Then $a u y=0$ and so $A_{k+1} \cap a u A_{k}=(0)$. For each $k \geqslant 1$ choose a right ideal $X_{k}$ maximal subject to $A_{k+1} \cap X_{k}=(0)$. Then $A_{k+1}+X_{k}$ is module-essential and hence essential in $N$. Writing $C_{k}=A_{k} \cap X_{k}$ we can find, since $l\left(A_{k}\right) \neq l\left(A_{k+1}\right)$, an $N$-subgroup $B_{k} \subset A_{k}$ with $B_{k} \neq(0)$ and $A_{k+1} \cap B_{k}=(0)$. Let $b \in B_{k} \cap\left(A_{k+1}+X_{k}\right)$ with $b \neq 0$. Then $b=t+x, t \in A_{k+1}, x \in X_{k}$ so $x=-t+b \in A_{k} \cap X_{k}$ and so $A_{k} \cap X_{k} \neq(0)$. It follows that 
$C_{k}$ is a non-zero right ideal of $N$ and $C_{k} \cap A_{k+1}=(0)$. We then have a chain $C_{1} \nsubseteq C_{1} \oplus C_{2} \mp \ldots$ which must terminate and so $N$ has the minimum condition on right annihilators.

Corollary 6. If $N$ is a regular d.g. near-ring in which module-essential $N$ subgroups are essential and if $N$ has the maximum condition on right annihilators and no infinite direct sums of right ideals then $N$ is a finite direct sum of ideals which are either rings of matrices over division rings or non-rings of the form $M_{G}(\Gamma)$ for a suitable type-2 $N$-module $\Gamma$.

Obviously Theorem 7 holds when $N$ is a regular near-ring in which module essential $N$-subgroups are essential and $N$ has the maximum condition on $N$ subgroups. In this case the conclusion of Corollary 6 again follows.

\title{
REFERENCES
}

(1) J. C. Beidleman, A note on regular near-rings, J. Ind. Math. Soc. 33 (1969), 207-210.

(2) G. BETSCH, Some Structure theorems on 2-primitive near-rings, Colloquia Mathematica Societatis János Bolyai: Rings, Modules and Radicals, Keszthely (Hungary) 1971 (North Holland, 1973).

(3) A. Frohlich, Distributively generated near-rings ( $I$ Ideal theory), Proc. London Math. Soc. (3) 8 (1958), 76-94.

(4) F. Hanson, One-sided prime ideals, Pac. J. Math. 58 (1975), 79-85.

(5) H. E. HeAtherLy, Regular near-rings, J. Ind. Math. Soc. 38 (1974), 345-364.

(6) К. Кон, On von Newmann regular rings, Canad. Math. Bull. 17 (1974), 283-284.

(7) S. Ligh, On division near-rings, Canad. J. Math. 21 (1969), 1366-1371. 187-197.

(8) A. Oswald, Completely reducible near-rings, Proc. Edinburgh Math. Soc. 20 (1977),

(9) G. PILZ, Near-rings (North Holland, 1977).

(10) P. N. Stewart, Semisimple radical classes, Pac. J. Math. 32 (1970), 249-254.

(11) G. SzETo, On regular near-rings with no non-zero nilpotent elements, Math. Jap. 79 (1974), 65-70.

\author{
Department of Mathematics and Statistics \\ TEESSIDE POLYTECHNIC \\ MidDlesBROUGH \\ Cleveland
}

